\title{
Physical illness and disability among elderly people in England and Wales: the Medical Research Council cognitive function and ageing study
}

\author{
C J Parker, K Morgan, M E Dewey and the Analysis Group of the MRC CFA study
}

\begin{abstract}
Study objective-This study was conducted as part of the MRC cognitive function and ageing study. It aimed to estimate the lifetime prevalence of self reported physical illnesses and other health related events, and the prevalence of limiting disability in people over 65 in six areas of England and Wales.

Design-Screening phase of a two stage prevalence study.

Setting-Geographically delimited areas in four urban and two rural areas including institutions.

Participants-Random population samples of people in their 65th year and above on the sample definition date, interviewed between 1989 and 1994. In Newcastle, Nottingham, and Oxford (urban) and in Cambridgeshire and Gwynedd(rural), the sample was stratified to provide equal numbers in the 65-74 and 75 years and over age groups. In Liverpool (urban), equal numbers in the five year age groups were taken.
\end{abstract}

Main results-Age standardised prevalences were calculated for each geographical area, sex, and age group (65-74, 75+). Many conditions were more prevalent in the older age group including stroke, Parkinson's disease, arthritis, diabetes, and shingles but hypertension was more common in the younger age group. Conditions that were more prevalent in men included angina, heart attack, stroke, head injury, and peptic ulcers while hypertension, shingles, pernicious anaemia, and thyroid disease were more common in women. There was a complex pattern of area differences for individual conditions. Cambridgeshire had generally low prevalences for many diseases, including vascular problems, Gwynedd and Newcastle had less healthy elderly populations, and Nottingham and Newcastle had the highest percentages of housebound.

Conclusions-This study provides the most robust available estimates for lifetime prevalence of a variety of health conditions on a regional and national basis. It shows the greatly increased prevalence of disability in the very old population, particularly women.

$(\Im$ Epidemiol Community Health 1997;51:494-501)
As the number of elderly people in the population rises there is an increasing need for detailed information on morbidity and disability in later life. In particular, the larger numbers of the very old will have major implications for resources in the early 21 st century: this age group has the highest morbidity rates and suffers more than any other from substantial co-morbidity.

In Britain, most of the information currently available in this area has been obtained either from large national surveys or from smaller, localised research initiatives. National studies such as the Office of Population Censuses and Surveys ${ }^{1}$ general household survey ${ }^{1}$ and, since 1991 , the decennial population census ${ }^{2}$ provide national data on broadly defined disabling long term illness and some evidence of regional variation in this. However, they offer few insights into the nature or distribution of underlying disorders or the extent of comorbidity among the very elderly. On the other hand, a number of studies, such as those recently completed in Leicestershire, ${ }^{3}$ Nottingham, ${ }^{4}$ and London, ${ }^{5}$ have provided detailed information on symptoms and levels of disability among elderly people living in specific areas. The use of national data to make estimates at a local level is difficult without a greater degree of detail and more information on regional differences. The extent to which results from local studies can be interpreted as reflecting regional variation is limited by restricted sample sizes and differences in survey methodology.

Data collected by the Medical Research Council cognitive function and ageing study (MRC CFAS) provide the opportunity to combine national coverage and evidence of regional variation with a high degree of detail on key disorders contributing to disability in later life. Since a major aim of the study was to evaluate possible risk factors for dementing illness, the screening interview collected information at baseline on a number of conditions which have been considered of importance in the aetiology of dementia. These factors were selected both by reviews of the literature (for example ${ }^{6}$ ) and from the consensus among European collaborators in the EURODEM incidence programme, ${ }^{7}$ and included a number of items on physical illness and disability. This information, collected from more than 17000 people living in six geographically diverse loca- 
tions in England and Wales, provides robust estimates for lifetime prevalence of a variety of health conditions as well as current levels of disability among the old and the very old. It is presented in this paper with particular emphasis on regional variations, differences in health problems among older and younger cohorts, and levels of co-morbidity.

\section{Methods}

DESIGN

CFAS is a longitudinal, two wave, two stage population based study of those aged 65 years and over in six centres throughout England and Wales. Urban populations were sampled in Liverpool, Newcastle, Nottingham, and Oxford and rural populations in Cambridgeshire and Gwynedd. All centres except Liverpool used identical methodology, which is described in detail in the first paper from the MRC CFAS $^{8}$; the study design used in Liverpool differed slightly and has been reported in full elsewhere. ${ }^{9}$ Briefly, in each centre, except Liverpool, a random sample was selected of sufficient size to produce 2500 interviews from individuals aged 65 years and over living within a defined geographical area, with equal numbers in the 65-74 and over 75 years age groups. In Liverpool, a total sample of over 5000 was selected with equal numbers in each 5 year age group for each sex. In all centres except Gwynedd, samples were drawn from family health services authority lists; in Gwynedd the sample was selected directly from general practitioner records. Elderly people living in residential accommodation and registered with a GP were included in the sample. An examination of long stay hospitals in each centre showed that in no case would the exclusion of any individuals not registered with a GP have more than a marginal effect on prevalence estimates.

\section{THE INTERVIEW}

The initial screening interview, which forms the basis of this paper, was conducted between 1991 and 1994 on the whole sample in all centres except Liverpool and contained items on sociodemographic variables, cognitive function, activities of daily living, and health factors, including a suggested risk set for dementia. ${ }^{7}$ Interviewers were also prompted to rate respondents on such things as mobility, sensory impairments, and demeanour during the interview. In Liverpool, interviewing was carried out between 1989 and 1991 while the interview schedule for the other five centres was being finalised. Although it shared many items, the interview used for the first half of the Liverpool sample included fewer questions on physical health than the eventual CFAS schedule. Some health questions were added to the Liverpool interview for the second half of the sample, which is itself a stratified random sample from the population with the same structure as the full sample. The appendix details the interview schedule items used for each health problem or event and indicates
KEY POINTS

- Self report of physical health and disability in older people shows the expected greater prevalence in the very elderly group for many, but not all, conditions.

- Differences between men and women do exists but are evenly balanced.

- Differences between centres suggest that reliance on national data or data from another location may be misleading.

where different wording was used in the Liverpool schedule. The five centre screening interview was translated into colloquial Welsh for those respondents in Gwynedd for whom this was the first language.

In all centres the interviews were carried out in respondents' homes using lap top computers which gave full wording for each question, enabled inappropriate sections to be skipped automatically, and prompted for immediate entry of valid responses. Inaccurate or absent responses to questions in the orientation section at the beginning of the interview caused an automatic engagement of "priority mode" in which only essential memory and cognition items together with interviewer ratings were completed. This mode could also be selected by the interviewer if the respondent was too frail or distressed to complete the interview. Data on self reported health factors are not available for these respondents. The interviewers were recruited locally but training and quality control procedures were implemented by the national coordinator of the study to ensure comparability between centres. The interviewing process is fully described in the first paper from the MRC CFAS. $^{8}$

\section{ANALYSIS}

Derived variables were produced to combine illnesses so that comparison with other existing data sets could be made. The vascular illnesses were combined after the style of the OPCS health survey for England. ${ }^{10}$ Current disability was calculated from the only three variables directly rated in that way: eyesight, hearing and being housebound. All prevalence rates were age standardised to the 1991 census of England and Wales. ${ }^{2}$

In a study of this size any difference which is of clinical importance will almost certainly be statistically significant, and many differences will be statistically significant without having a clinical or scientific importance. For this reason statistical testing has been kept to a minimum in this paper. In the tables, data are presented for comparative purposes from the major studies which have estimated prevalences for these conditions. Again, even for a moderate sample, any difference which might be of practical importance will almost certainly be statistically significant. For instance, an illness with a prevalence of $20 \%$ in a sample of the size of the CFAS would be seen as statistically significant from the prevalence of $23 \%$ in a sample of 1000 , and the statistically significant difference would be even smaller if the second study were larger. 
Table 1 Prevalence (\%) of self reported vascular events and disease in each centre (see Appendix for definitions)

\begin{tabular}{|c|c|c|c|c|c|c|c|c|c|c|c|c|c|c|c|c|}
\hline \multirow[b]{2}{*}{ Age group (y) } & \multicolumn{2}{|c|}{ Cambridgeshire } & \multicolumn{2}{|c|}{ Gwynedd } & \multicolumn{2}{|c|}{ Liverpool } & \multicolumn{2}{|c|}{ Newcastle } & \multicolumn{2}{|c|}{ Nottingham } & \multicolumn{2}{|l|}{ Oxford } & \multicolumn{2}{|c|}{ CFAS overall ${ }^{\star}$} & \multicolumn{2}{|c|}{ oPCSt } \\
\hline & $65-74$ & $75+$ & $65-74$ & $75+$ & $65-74$ & $75+$ & $65-74$ & $75+$ & $65-74$ & $75+$ & $65-74$ & $75+$ & $65-74$ & $75+$ & $65-74$ & $75+$ \\
\hline \multicolumn{17}{|c|}{ High blood pressure } \\
\hline M & 31.1 & 23.9 & 33.6 & 28.1 & 27.2 & 21.0 & 31.6 & 22.8 & 32.1 & 24.1 & 30.1 & 24.6 & 31.6 & 24.9 & 32 & 25 \\
\hline $\mathrm{F}$ & 34.2 & 32.5 & 40.8 & 37.7 & 30.9 & 28.0 & 33.9 & 32.6 & 32.7 & 32.3 & 41.1 & 32.9 & 36.5 & 33.6 & 34 & 28 \\
\hline \multicolumn{17}{|l|}{ Angina } \\
\hline M & 12.2 & 15.1 & 20.9 & 15.2 & & & 18.6 & 19.1 & 12.7 & 13.9 & 14.5 & 13.3 & 15.6 & 15.2 & 13 & 16 \\
\hline $\mathrm{F}$ & 6.4 & 12.5 & 10.3 & 14.0 & & & 11.9 & 17.4 & 10.0 & 13.0 & 10.1 & 13.7 & 9.8 & 14.1 & 9 & 12 \\
\hline \multicolumn{17}{|l|}{ Heart attack } \\
\hline M & 12.2 & 11.4 & 17.4 & 14.8 & 14.4 & 13.9 & 16.3 & 15.4 & 12.8 & 15.3 & 14.6 & 14.3 & 14.5 & 14.1 & 8 & 10 \\
\hline F & 4.6 & 7.5 & 7.5 & 9.0 & 6.7 & 7.9 & 5.8 & 10.5 & 5.4 & 9.1 & 6.5 & 8.1 & 6.0 & 8.8 & 5 & 7 \\
\hline \multicolumn{17}{|l|}{ Stroke } \\
\hline$M$ & 5.7 & 8.7 & 5.1 & 10.2 & & & 6.5 & 12.4 & 8.4 & 12.4 & 6.1 & 9.2 & 6.4 & 10.5 & 3 & 6 \\
\hline F & 2.8 & 7.1 & 5.3 & 6.8 & & & 4.6 & 9.7 & 5.2 & 8.8 & 3.6 & 7.9 & 4.3 & 8.1 & 2 & 7 \\
\hline \multicolumn{17}{|c|}{ Intermittent claudication } \\
\hline M & 2.0 & 3.9 & 6.0 & 3.9 & & & 1.6 & 2.7 & 1.3 & 0.5 & 1.3 & 2.3 & 2.4 & 2.7 & & \\
\hline $\mathrm{F}$ & 3.4 & 2.2 & 2.4 & 3.3 & & & 0.9 & 1.1 & 0.1 & 0.3 & 1.7 & 1.8 & 1.7 & 1.7 & & \\
\hline
\end{tabular}

Cognitive function and ageing study (CFAS) rates age standardised to 1991 census (England and Wales). ${ }^{2}$

$\star$ Overall rate for five centres (excluding Liverpool as questions differed).

†Office of Population Censuses and Surveys (OPCS) health survey for England $1991^{10}$ (private households only).

Table 2 Prevalence (\%) of summary measures of cardiovascular disease and related conditions in each centre (see Appendix for definitions)

\begin{tabular}{|c|c|c|c|c|c|c|c|c|c|c|c|c|c|c|}
\hline \multirow{2}{*}{$\begin{array}{l}\text { Age group } \\
\text { (y) }\end{array}$} & \multicolumn{2}{|c|}{ Cambridgeshire } & \multicolumn{2}{|c|}{ Gwynedd } & \multicolumn{2}{|c|}{ Newcastle } & \multicolumn{2}{|c|}{ Nottingham } & \multicolumn{2}{|l|}{ Oxford } & \multicolumn{2}{|c|}{$\begin{array}{l}\text { CFAS } \\
\text { overall * }\end{array}$} & \multicolumn{2}{|c|}{ oPCSt } \\
\hline & $65-74$ & $75+$ & $65-74$ & $75+$ & $65-74$ & $75+$ & $65-74$ & $75+$ & $65-74$ & $75+$ & $65-74$ & $75+$ & $65-74$ & $75+$ \\
\hline \multicolumn{15}{|c|}{ Heart attack or stroke } \\
\hline $\mathrm{M}$ & 17.3 & 17.0 & 20.6 & 23.0 & 20.7 & 26.2 & 19.2 & 25.0 & 18.7 & 21.4 & 19.3 & 22.3 & 15 & 16 \\
\hline F & 7.3 & 13.5 & 11.9 & 15.1 & 10.1 & 17.9 & 10.5 & 16.5 & 9.7 & 14.9 & 9.9 & 15.6 & 8 & 12 \\
\hline \multicolumn{15}{|c|}{ Angina, but not heart attack or stroke } \\
\hline $\mathrm{M}$ & 5.4 & 7.5 & 8.5 & 6.2 & 7.7 & 9.1 & 5.8 & 6.3 & 5.8 & 5.9 & 6.6 & 6.9 & 6 & 11 \\
\hline $\mathrm{F}$ & 3.6 & 6.9 & 4.6 & 8.5 & 7.6 & 9.4 & 5.7 & 6.7 & 5.2 & 7.9 & 5.4 & 7.9 & 6 & 6 \\
\hline \multicolumn{15}{|c|}{ Only hypertension or diabetes } \\
\hline $\mathrm{M}$ & 23.5 & 21.8 & 21.6 & 19.2 & 21.2 & 12.3 & 21.8 & 17.6 & 22.2 & 20.3 & 22.1 & 18.6 & 24 & 19 \\
\hline $\mathrm{F}$ & 30.2 & 25.6 & 31.6 & 28.6 & 26.7 & 21.6 & 25.8 & 25.7 & 32.7 & 24.3 & 29.4 & 25.1 & 30 & 25 \\
\hline \multicolumn{15}{|c|}{ None of the above conditions } \\
\hline M & 53.8 & 53.5 & 49.3 & 51.5 & 50.3 & 52.3 & 53.2 & 51.0 & 53.3 & 52.3 & 52.1 & 52.1 & 56 & 54 \\
\hline $\mathrm{F}$ & 58.9 & 54.0 & 51.9 & 47.8 & 55.6 & 51.1 & 58.0 & 51.0 & 52.3 & 52.9 & 55.3 & 51.4 & 56 & 57 \\
\hline
\end{tabular}

Cognitive function and ageing study (CFAS) rates age standardised to 1991 census (England and Wales). ${ }^{2}$

*Overall rate for five centres (Liverpool data not available for all conditions).

†Office of Population Censuses and Surveys (OPCS) health survey for England $1992^{10}$ (private households only).

Table 3 Prevalence (\%) of self reported cerebral events and disease in each centre (see appendix for definitions)

\begin{tabular}{|c|c|c|c|c|c|c|c|c|c|c|c|c|c|c|}
\hline \multirow{2}{*}{$\begin{array}{l}\text { Age group } \\
\text { (y) }\end{array}$} & \multicolumn{2}{|c|}{ Cambridgeshire } & \multicolumn{2}{|c|}{ Gwynedd } & \multicolumn{2}{|c|}{ Liverpool } & \multicolumn{2}{|c|}{ Newcastle } & \multicolumn{2}{|c|}{ Nottingham } & \multicolumn{2}{|l|}{ Oxford } & \multicolumn{2}{|c|}{$\begin{array}{l}\text { CFAS } \\
\text { overall }^{*}\end{array}$} \\
\hline & $65-74$ & $75+$ & $65-74$ & $75+$ & $65-74$ & $75+$ & $65-74$ & $75+$ & $65-74$ & $75+$ & $65-74$ & $75+$ & $65-74$ & $75+$ \\
\hline \multicolumn{15}{|c|}{ Head injury } \\
\hline $\mathrm{M}$ & 20.7 & 18.6 & 13.7 & 12.6 & 13.9 & 12.4 & 19.3 & 14.0 & 19.4 & 15.9 & 15.8 & 15.0 & 17.9 & 15.3 \\
\hline $\mathrm{F}$ & 8.8 & 6.9 & 7.0 & 5.8 & 3.9 & 4.6 & 8.2 & 8.5 & 7.4 & 7.8 & 9.6 & 7.6 & 8.2 & 7.3 \\
\hline \multicolumn{15}{|l|}{ Seizures } \\
\hline M & 1.7 & 1.0 & 1.1 & 1.3 & 1.9 & 1.5 & 2.4 & 1.4 & 1.1 & 1.2 & 2.4 & 0.8 & 1.7 & 1.1 \\
\hline F & 1.8 & 1.9 & 1.9 & 1.4 & 2.3 & 0.9 & 2.4 & 1.6 & 1.0 & 0.9 & 0.9 & 1.4 & 1.6 & 1.5 \\
\hline \multicolumn{15}{|c|}{ Meningitis/encephalitis } \\
\hline M & $0.6^{\circ}$ & 0.2 & 1.3 & 1.0 & 1.2 & 1.5 & 2.5 & 1.6 & 1.2 & 1.3 & 1.3 & 1.4 & 1.4 & 1.1 \\
\hline F & 0.0 & 0.1 & 1.1 & 0.9 & 1.2 & 0.9 & 0.6 & 0.7 & 1.2 & 0.5 & 1.7 & 0.7 & 0.9 & 0.6 \\
\hline \multicolumn{15}{|c|}{ Parkinson's disease } \\
\hline M & 0.5 & 2.2 & 0.1 & 1.4 & 0.7 & 0.7 & 0.5 & 1.6 & 1.0 & 2.5 & 1.1 & 1.2 & 0.7 & 1.8 \\
\hline $\mathrm{F}$ & 1.0 & 0.4 & 0.8 & 1.3 & 0.2 & 0.6 & 0.0 & 1.7 & 0.8 & 1.1 & 0.5 & 1.7 & 0.6 & 1.2 \\
\hline
\end{tabular}

Cognitive function and ageing study (CFAS) rates age standardised to 1991 Census (England and Wales). ${ }^{2}$

$\star$ Overall rate for five centres (excluding Liverpool as some questions differed).

Tables 1-5 show that a typical prevalence rate in an individual centre of $10 \%$ has a $95 \%$ confidence interval $(95 \% \mathrm{CI})$ of $7.3 \%, 12.7 \%$, and a typical, five centre overall prevalence rate of $10 \%$ has a $95 \% \mathrm{CI}$ of $8.7 \%, 11.3 \%$.

Because of the longitudinal nature of the CFAS, versions of the research database are released at intervals: the results reported here are based on version 3 released in October 1995.

Results

In the five centres excluding Liverpool, a screening interview was achieved for 13009 respondents $-80 \%$ of those approached. There are 20 conditions to be considered in the remainder of this paper; $12368(95.1 \%)$ of the respondents in the five centres have data on all of these. The remainder are fairly evenly split between those who had 1 or 2 missing (245; $1.9 \%$ ) and those who had 2 or fewer present $(320 ; 2.5 \%)$ with a small number with intermediate numbers missing $(76 ; 0.6 \%)$. After excluding those people who did not provide useful data on at least three illnesses, the numbers remaining in each centre were Cambridgeshire 2569, Gwynedd 2556, Newcastle 2446, 
Table 4 Prevalence (\%) of other self reported disease in each centre (see Appendix for definitions)

\begin{tabular}{|c|c|c|c|c|c|c|c|c|c|c|c|c|c|c|c|c|}
\hline \multirow[b]{2}{*}{ Age group (y) } & \multicolumn{2}{|c|}{ Cambridgeshire } & \multicolumn{2}{|c|}{ Gwynedd } & \multicolumn{2}{|c|}{ Liverpool } & \multicolumn{2}{|c|}{ Newcastle } & \multicolumn{2}{|c|}{ Nottingham } & \multicolumn{2}{|l|}{ Oxford } & \multicolumn{2}{|c|}{ CFAS overall ${ }^{\star}$} & \multicolumn{2}{|c|}{ OPCSt } \\
\hline & $65-74$ & $75+$ & $65-74$ & $75+$ & $65-74$ & $75+$ & $65-74$ & $75+$ & $65-74$ & $75+$ & $65-74$ & $75+$ & $65-74$ & $75+$ & $65-74$ & $75+$ \\
\hline \multicolumn{17}{|l|}{ Arthritis } \\
\hline M & 37.0 & 44.7 & 41.2 & 41.3 & & & 46.6 & 47.1 & 42.8 & 46.1 & 34.1 & 40.8 & 40.2 & 44.0 & & \\
\hline $\mathrm{F}$ & 52.7 & 60.2 & 52.3 & 57.7 & & & 59.7 & 61.9 & 56.2 & 59.9 & 50.9 & 60.5 & 54.3 & 60.1 & & \\
\hline \multicolumn{17}{|l|}{ Shingles } \\
\hline$M$ & 18.3 & 26.6 & 20.8 & 24.7 & 15.6 & 21.6 & 20.7 & 24.9 & 17.5 & 23.7 & 16.9 & 27.9 & 18.7 & 25.6 & & \\
\hline $\mathrm{F}$ & 23.7 & 30.0 & 29.6 & 29.6 & 18.8 & 26.6 & 22.0 & 27.3 & 20.6 & 25.3 & 21.9 & 26.7 & 23.5 & 27.8 & & \\
\hline M & 10.5 & 11.9 & 14.8 & 16.8 & & & 16.8 & 22.4 & 15.9 & 15.6 & 12.9 & 15.6 & 14.1 & 16.3 & & \\
\hline F & 6.3 & 6.7 & 7.4 & 7.8 & & & 9.2 & 9.2 & 6.1 & 5.1 & 7.2 & 9.1 & 7.2 & 7.6 & & \\
\hline \multicolumn{17}{|l|}{ Bronchitis } \\
\hline M & 14.8 & 17.2 & 10.5 & 11.4 & & & 18.6 & 17.7 & 11.2 & 15.1 & 9.5 & 7.8 & 12.9 & 13.6 & & \\
\hline F & 12.5 & 13.0 & 12.9 & 12.1 & & & 18.0 & 14.3 & 14.2 & 11.4 & 10.6 & 8.9 & 13.6 & 11.9 & & \\
\hline \multicolumn{17}{|l|}{ Asthma } \\
\hline M & 10.4 & 10.9 & 10.8 & 9.0 & & & 10.6 & 8.9 & 7.9 & 7.2 & 10.7 & 11.4 & 10.1 & 9.5 & & \\
\hline $\mathrm{F}$ & 9.2 & 6.9 & 9.6 & 7.8 & & & 10.5 & 8.0 & 12.1 & 7.6 & 12.1 & 7.7 & 10.7 & 7.6 & & \\
\hline \multicolumn{17}{|c|}{ Thyroid disease } \\
\hline \multicolumn{17}{|l|}{ Diabetes } \\
\hline M & 6.0 & 10.8 & 7.3 & 7.9 & & & 5.3 & 5.1 & 5.8 & 7.9 & 4.9 & 8.2 & 5.9 & 8.1 & 5 & 4 \\
\hline $\mathrm{F}$ & 5.7 & 7.5 & 3.0 & 5.1 & & & 3.5 & 3.6 & 5.3 & 8.4 & 5.4 & 5.5 & 4.6 & 6.0 & 6 & 7 \\
\hline \multicolumn{17}{|c|}{ Pernicious anaemia } \\
\hline M & 0.3 & 1.5 & 0.8 & 2.6 & & & 0.8 & 2.4 & 1.2 & 2.8 & 0.4 & 1.4 & 0.7 & 2.2 & & \\
\hline $\mathrm{F}$ & 1.8 & 2.0 & 3.9 & 6.3 & & & 3.4 & 4.4 & 4.3 & 5.4 & 4.2 & 2.9 & 3.5 & 4.2 & & \\
\hline
\end{tabular}

Cognitive function and ageing study (CFAS) rates age standardised to 1991 Census (England and Wales). ${ }^{2}$

*Overall rate for five centres (excluding Liverpool as some questions differed).

†Office of Population Censuses and Surveys (OPCS) health survey for England 1992 (private households only). ${ }^{10}$

Table 5 Prevalence (\%) of interviewer rated disabilities in each centre (see Appendix for definitions)

\begin{tabular}{|c|c|c|c|c|c|c|c|c|c|c|c|c|c|c|}
\hline \multirow[b]{2}{*}{ Age group (y) } & \multicolumn{2}{|c|}{ Cambridgeshire } & \multicolumn{2}{|c|}{ Gwynedd } & \multicolumn{2}{|c|}{ Newcastle } & \multicolumn{2}{|c|}{ Nottingham } & \multicolumn{2}{|l|}{ Oxford } & \multicolumn{2}{|c|}{ CFAS overall $\neq$} & \multicolumn{2}{|c|}{ Other source } \\
\hline & $65-74$ & $75+$ & $65-74$ & $75+$ & $65-74$ & $75+$ & $65-74$ & $75+$ & $65-74$ & $75+$ & $65-74$ & $75+$ & $65-74$ & $75+$ \\
\hline \multicolumn{15}{|c|}{ Eyesight -marked problem or blind } \\
\hline $\mathrm{M}$ & 0.8 & 4.5 & 0.3 & 5.7 & 0.4 & 3.1 & 0.5 & 4.0 & 1.5 & 6.4 & 0.7 & 4.8 & & \\
\hline $\mathrm{F}$ & 0.6 & 6.2 & 0.8 & 7.0 & 1.0 & 5.0 & 1.5 & 7.0 & 1.0 & 9.2 & 1.0 & 6.9 & & \\
\hline \multicolumn{15}{|c|}{ Hearing - marked problem or deaf } \\
\hline $\mathrm{M}$ & 0.7 & 7.6 & 0.6 & 4.1 & 0.3 & 2.9 & 0.7 & 4.8 & 2.5 & 8.1 & 1.0 & 5.5 & & \\
\hline $\mathrm{F}$ & 0.4 & 5.8 & 0.5 & 3.9 & 0.0 & 4.6 & 0.6 & 4.2 & 1.8 & 6.8 & 0.7 & 5.1 & & \\
\hline $\mathrm{M}$ & 2.5 & 6.4 & 2.5 & 9.5 & 4.1 & 10.2 & 4.4 & 13.5 & 2.6 & 10.8 & 3.2 & 10.1 & $4^{\star}$ & $7^{\star}$ \\
\hline $\mathrm{F}$ & 3.3 & 17.7 & 3.8 & 23.1 & 5.9 & 22.9 & 4.0 & 24.9 & 4.0 & 21.8 & 4.2 & 22.1 & $7^{\star}$ & $16^{\star}$ \\
\hline \multicolumn{15}{|c|}{ At least one of the above } \\
\hline M & 3.5 & 15.3 & 3.1 & 16.2 & 4.9 & 13.0 & 5.1 & 17.9 & 6.0 & 20.2 & 4.5 & 16.6 & $8.7 \dagger$ & $25.0 \dagger$ \\
\hline $\mathrm{F}$ & 4.0 & 23.6 & 4.5 & 26.1 & 6.5 & 27.0 & 5.5 & 29.3 & 6.5 & 28.1 & 5.5 & 26.9 & $10.6 \dagger$ & $36.9 \dagger$ \\
\hline \multicolumn{15}{|c|}{ Census - limiting long-term illness $\S$} \\
\hline M & 30.0 & 43.1 & 35.5 & 47.7 & 41.0 & 51.1 & 37.9 & 51.0 & 31.8 & 48.9 & 34.9 & 48.4 & & \\
\hline $\mathrm{F}$ & 25.6 & 49.2 & 28.7 & 53.2 & 35.9 & 58.4 & 34.6 & 57.3 & 30.0 & 52.4 & 31.8 & 55.6 & & \\
\hline \multicolumn{15}{|c|}{ None of the 20 conditions in Tables 1 to 5} \\
\hline M & 18.2 & 8.8 & 15.1 & 13.8 & 12.8 & 11.4 & 16.1 & 11.9 & 18.9 & 10.9 & 16.3 & 11.3 & & \\
\hline
\end{tabular}

Cognitive function and ageing study (CFAS) rates age standardised to 1991 census (England and Wales). ${ }^{2}$

*Health and lifestyle survey (private households only). ${ }^{11}$

tOffice of Population Censuses and Surveys (OPCS) survey of disability in Great Britain —severity levels 5-10 (age groups 60-74, 75 +). ${ }^{13}$

$\ddagger$ Overall rate for five centres (Liverpool data not available for these conditions).

§County reports data, 1991 census of England and Wales. ${ }^{2}$

Nottingham 2452, and Oxford 2666. These can be taken as the effective base for the percentages for individual self-reported conditions, although the actual bases vary from these by up to $0.8 \%$.

In Liverpool, 5222 respondents were interviewed, a response rate of $87 \%$. Base numbers vary because of the introduction of some questions in the second year: for four conditions (meningitis/encephalitis, Parkinson's disease, seizures and shingles) the effective base is 2470 with the actual bases varying from this by up to $0.2 \%$, and for the remainder 5060 varying by up to $1.3 \%$.

The 20 conditions to be reported have been divided into four groups: vascular events and disease, cerebral events and disease, other medical conditions, and disability. Table 1 presents the first of these: the prevalence of various self reported vascular problems, with the conditions presented in descending order of overall prevalence. Self reported lifetime high blood pressure was more prevalent in women than in men for all centre/age groups and was also consistently more prevalent in the younger age group for both sexes. Comparison of the four age/sex rates for individual centres with the overall CFAS figures shows that Cambridgeshire and Liverpool have relatively low prevalences and Gwynedd relatively high. Angina was more prevalent in men than women in all but one centre/age group. Among women it was consistently more prevalent in the older age group but there was no clear age related pattern for men. Cambridgeshire had relatively low angina rates and Newcastle relatively high ones. Heart attack was also more prevalent in men than women and, like angina, had higher prevalences for older rather than younger women. However, among men, the 
younger age group had higher rates than the older in all centres except Nottingham. Cambridgeshire again was relatively low in prevalence and Gwynedd was the only centre in which all four age/sex groups had higher heart attack rates than CFAS overall. Stroke was similar to angina and heart attack in being more prevalent in men than women, and was also consistently more prevalent in the older age group. Cambridgeshire had another relatively low set of rates for this condition as did Oxford, with Newcastle and Nottingham relatively high. Intermittent claudication, a condition with low prevalence compared to the previous four, showed no clear age or sex patterns but was relatively more prevalent in Gwynedd and less in Nottingham, with quite large differences between these two.

The comparison data set shown in the final column of table 1 is from the OPCS health survey for England. ${ }^{10}$ These figures are broadly comparable with the CFAS figures, although for heart attack and stroke the CFAS figures are rather higher. The OPCS study was conducted in private households only, whereas CFAS included people living in sheltered accommodation, nursing homes, and other institutions.

Table 2 shows the prevalences of the same conditions arranged in the hierarchical scheme used in the OPCS study. People who provided valid data on at least one of the five conditions were included in this summary measure, giving a base equal to the effective base for individual conditions. This confirms the higher rates of more severe vascular disease among men and among the older age group, and shows that Newcastle had relatively high rates of severe disease and Cambridgeshire and Oxford relatively low rates, although centre differences were not large.

Table 3 shows the prevalence of self report of a number of conditions associated with cerebral disease and injury. Head injury is shown to be predominantly an event affecting men, with rates around double those for women The younger group of men has consistently higher rates than the older group. There is no clear pattern across the centres for head injury, although Gwynedd and Liverpool have slightly lower rates than the study overall. Seizures show no clear age related or sex related patterns, and the only centre appearing somewhat out of line is Newcastle with slightly higher rates. For meningitis/encephalitis the only noticeable feature is a relatively low set of prevalences for Cambridgeshire. Parkinson's disease is more prevalent in the older age group with no sex or centre patterns.

Table 4 shows the prevalences of a variety of other medical conditions which were also investigated. Arthritis is consistently more prevalent among women and also among the older age group, and has slightly higher rates in Newcastle than in the other centres. Shingles is more prevalent in women and also in the older age group. It has slightly lower rates in Liverpool and Nottingham. Peptic ulcers are shown to be more prevalent in men and have their highest rates in Newcastle and Gwynedd and their lowest in Cambridgeshire. Bronchitis rates show no age or sex pattern but are relatively high in Newcastle and low in Oxford. Asthma is more prevalent in younger than in older women with no clear age pattern for men. Oxford has somewhat high rates for this condition. Thyroid disease shows the largest sex difference of any of the conditions reported here, with female prevalences of the order of five times higher than those for males. The highest rates are in Gwynedd and the lowest in Liverpool. Diabetes is more prevalent in the older age group and, among centres, is highest in Cambridgeshire and lowest in Newcastle. The figures shown from the OPCS study for this condition are broadly comparable with the overall CFAS figures, although they suggest slightly higher female prevalences. Pernicious anaemia is shown to be more prevalent in women and also in the older age group. Rates for pernicious anaemia are lowest in Cambridgeshire and highest in Gwynedd and Nottingham.

Table 5 shows firstly the prevalence of three important disability variables: these were not collected as self report in the same way as the other conditions, but are based on interviewer ratings. For hearing, a positive rating indicates that hearing was a marked problem during the interview, irrespective of whether an aid was worn, and similarly for eyesight and spectacles. These two variables therefore indicate disability in these areas rather than simple impairment of faculties. All of these three disabilities have markedly higher prevalences among the older age group, with the differentials being higher for women than for men. So, for example, marked visual disability increases around fourfold in men and around sevenfold in women between the younger and older groups. Hearing disability is more prevalent in men than in women in all but one centre/age group, and being housebound is similarly more prevalent in women than in men. Among those aged over 75 years, the women are about twice as likely as men to be housebound.

Centre differences are by no means consistent across these three conditions. For eyesight problems Oxford tends to show higher rates, and for hearing problems Oxford is again high with Newcastle having the lowest rates. However, for being housebound there is an entirely different pattern, with Nottingham and Newcastle having high prevalences and Gwynedd low.

This table also shows prevalence rates for limitation by one or more of these three conditions. Whereas the percentages for the three individual disabilities are based on the number with valid data for that item, the prevalence of limitation by at least one of the conditions is based on the number with valid data for all three. Again, women have consistently higher rates than men and the older age group has rates some four to five times higher than the younger. By virtue of its higher rates for eyesight and hearing problems, Oxford comes highest for this summary measure with Cambridgeshire and Gwynedd relatively low. To compare the prevalence of "limiting long-term 
illness" from the 1991 census, county reports for each of the CFAS areas were tabulated. For this measure, Cambridgeshire shows a relatively low prevalence as it does in CFAS but the high prevalence centres are Newcastle and Nottingham.

The final information shown in table 5 is the percentage of people with none of the 17 self reported conditions or the three interviewerrated disabilities described above, based on the number with valid data for at least 15 of the 20 conditions. Unsurprisingly, this shows lower rates in the older age group: there is no clear cut pattern of either sex or area differences.

\section{Discussion}

There are relatively few comparable studies for monitoring the conditions reported in this paper. The only area for which there are several published reports is that of cardiovascular problems. The figures in tables 1 and 2 suggest that the OPCS study and CFAS are broadly similar. The main difference is the higher prevalence in CFAS of the more serious conditions (history of heart attack or stroke), probably reflecting the fact that the OPCS study sampled private households only, whereas CFAS also interviewed people living in institutions. The restriction of the OPCS study to England, whereas CFAS includes a Welsh centre, is unlikely to be important in these comparisons. Apart from this the age/sex differences found in the two studies do seem comparable.

For other conditions there is less opportunity to make simple comparisons. The prevalence of epilepsy is usually lower than the figures quoted in table 3 for history of seizures. For instance, Forsgren ${ }^{13}$ found prevalences of 0.6 and 0.4 (men 60-69 and 70+) and 0.6 and 0.3 (women) but these are for diagnosed epilepsy, whereas CFAS asks about a history of seizures.

Two studies of diabetes have found comparable age-sex specific figures. Huijsman ${ }^{14}$ found values of 5.6 and 5.9 (men, CFAS age bands) and 7.0 and 8.3 (women). In the US the National Diabetes Data Group found values of 8.8, 7.9 and 8.8, 9.5. ${ }^{15}$

CFAS decided that asking separately about osteoarthritis and rheumatoid arthritis would not be helpful, and that field diagnosis was also unlikely to be practicable. Because of this the results of comparisons with published data must be tentative. Radiological studies have shown changes indicative of osteoarthritis in almost $100 \%$ of the population at these ages, but symptoms are not universal. The usual finding of a higher prevalence in women is supported in CFAS.

Hearing difficulty presents a range of estimates based on objective audiological assessment and self reported difficulty. The CFAS estimates come close to the figures of Davis $e t$ $a l^{16}$ for great difficulty hearing in a quiet room (with best ear) which are $1.9 \%(61-70), 4.1 \%$ (71-80), 8.7\% (81-99). This does seem a comparable definition to the interviewer rating used in CFAS. By comparison the GHS ${ }^{1}$ gives much higher prevalences for self reported diffi- culty $-36 \%$ and $45 \%$ (men, CFAS age bands) and $21 \%$ and $41 \%$ (women).

It could be expected that the self reported prevalences presented here represent marginal underestimates of the true rates in the community. Just under $2.5 \%$ of the sample gave no valid data on the health section of the interview schedule, and these respondents were by definition the most frail, either physically, cognitively, or both. There is also some evidence that there may be a degree of under-reporting on some health questions by those respondents able to give a valid response. While there are no data to compare self report with medical records, a subsample of respondents was selected for more detailed assessment and in these a comparison can be made between self reported prevalences and rates obtained from a close relative or friend. In some cases this shows a slightly higher prevalence reported by the proxy. This is the subject of a further paper from the CFAS. ${ }^{17}$ The overall response rate for the study is also discussed in another paper. ${ }^{8}$ In brief, the refusal rate of $20 \%$ compares well with similar studies. Unusually for large scale community studies, the response rate was slightly higher in the oldest age groups, suggesting that the oldest and perhaps frailest are not differentially unrepresented in the sample.

Findings on co-morbidity with regard to combinations of vascular disease and related conditions have been presented here. The data on prevalence of "no illness or disability" also gives some indication that conditions do not occur singly in individuals. A more detailed analysis of patterns of co-morbidity in elderly people would be too lengthy to include here and will be covered in a further paper.

Prevalence rates for the 20 items reported in this paper have been compared on the basis of age, sex, and geographical area, and it may be helpful finally to summarise the findings under these three headings.

AGE

The results reported here show higher prevalence in the older age group for stroke, Parkinson's disease, arthritis, diabetes, pernicious anaemia, shingles, and most notably for the three disability measures. Additionally, older women show higher prevalences than younger women for angina and heart attack. The reverse situation of higher prevalence in the younger cohort has been found for high blood pressure, and also for heart attack and head injury in men and for asthma in women. Differences in the age specific prevalences of persisting conditions reflect the balance between incidence and differential mortality. Any such item which is more prevalent in the younger group either has a high differential mortality or a large cohort effect. Conversely, a condition higher in prevalence in the older age group is either protective or has a high incidence at or before that age group. We suggest that differential mortality probably explains the hypertension results, although an alternative explanation may be that older 
cohorts may have been less likely to be told of their hypertension.

SEX

With regard to the vascular disorders, men show higher prevalences for angina, heart attack, and stroke but women have higher rates for high blood pressure. The interviewers were instructed to exclude hypertension related to pregnancy, so this cannot be the explanation for the higher female prevalence. Perhaps the greater contact of women with health services has provided more scope for opportunistic screening for hypertension. Of the remaining conditions, ulcers, history of head injury, and hearing impairment are all more prevalent in men than in women. Female prevalences are substantially higher than those for males for thyroid disease and for being housebound, and are also higher for arthritis, pernicious anaemia, and shingles.

\section{GEOGRAPHICAL AREA}

On the basis of the results reported above, the study area with the healthiest elderly population is undoubtedly Cambridgeshire. Prevalences here are consistently lower than the figures for the study overall for all the main vascular conditions as well as peptic ulcers, meningitis/ encephalitis, and pernicious anaemia. The only condition for which Cambridgeshire has somewhat high rates is diabetes. The other rural area sampled, Gwynedd, shows a very different pattern with high prevalences for heart attack, high blood pressure, intermittent claudication, peptic ulcers, thyroid disease, and pernicious anaemia, and relatively low rates for head injury only. However, the prevalence of being housebound is the lowest in Gwynedd. For those conditions with data from the Liverpool centre, prevalences here are all lower than the overall CFAS figures. Newcastle seems to have a much less healthy elderly population with angina, stroke, seizures, arthritis, and peptic ulcers being of relatively high prevalence here. Nottingham has prevalence rates close to the CFAS mean for most conditions, with stroke and pernicious anaemia being relatively high and shingles and intermittent claudication relatively low. Nottingham and Newcastle have the highest rates for being housebound. Oxford has the lowest rates for stroke and high rates for asthma, eyesight problems, and hearing problems.

This rather complex pattern of disease vindicates the original decision to undertake a multicentre incidence study, and reinforces the suggestion that national level disability data may conceal important local level differences in patterns of late life morbidity. Certainly, the reported diseases and disabilities in tables 1-5 are not easily accounted for in terms either of north-south divisions or of urban-rural differences. With the increasing emphasis on balancing local health care provision with local health care need, such findings emphasise the value of exploring age, gender, and disease specific prevalences within clearly defined geographical areas.
Appendix: interview schedule items

SELF REPORTED LIFE TIME HEALTH EVENTS AND DISEASE

Angina: Have you ever suffered from angina? (Only recorded if diagnosed by a doctor)

Arthritis: Have you ever suffered from arthritis? (Including persistent joint pain)

Asthma: Have you ever suffered from asthma? (Recording whether childhood only)

Bronchitis: Have you ever suffered from chronic bronchitis?

Diabetes: Have you ever had sugar diabetes?

Head injury: Have you ever had a serious head injury and been unconscious after it? (Have you ever been knocked out?)

(Liverpool: Have you ever suffered a serious injury to the head? Did it make you lose consciousness?)

Heart attack: Have you ever suffered from a heart attack?

(Liverpool: Have you ever had a heart attack diagnosed by a doctor when several weeks rest was advised?)

High blood pressure: Have you ever been told that you have high blood pressure? (Excluding high blood pressure in pregnancy, recording whether told by GP or other)

(Liverpool: Have you ever had high blood pressure? (Only recorded if treated by hypotensive medication))

Intermittent claudication: Have you ever suffered from intermittent claudication? (Only recorded if diagnosed by a doctor)

Meningitis/encephalitis: Have you ever suffered from meningitis or encephalitis (brain fever)?

Parkinson's disease: Have you ever been diagnosed as having Parkinson's disease?

(Liverpool: Have you ever suffered from Parkinson's disease? (Recording whether treated or untreated - only included in this analysis if treated))

Peptic ulcers: Have you ever suffered from peptic ulcers? (gastric and duodenal)

Pernicious anaemia: Have you ever suffered from pernicious anaemia?

Seizures: Have you ever had fits or epilepsy? (Recording whether only one known fit or more than one fit - not included in this analysis if one only)

Shingles: Have you ever suffered from shingles? (Recording whether in the body or the head)

Stroke: Have you ever had a stroke that required medical attention? (Only recorded if episode lasted 48 hours or longer with partial paralysis, blindness, or speech disturbance.)

Thyroid problems: Have you ever suffered from thyroid problems? (Recording whether underactive or overactive, past or present)

(Liverpool: Have you ever suffered from an overactive or underactive thyroid? (Only recorded if diagnosed by a doctor))

\section{INTERVIEWER RATED DISABILITIES}

Eyesight: Did the subject have poor/no eyesight that interfered with reading, writing or drawing? (Recording whether to some extent, to marked extent, or blind - not included in this analysis if 'to some extent') 
Hearing: Did the subject have hearing problems that interfered with the questioning? (Recording whether to some extent, to marked extent or deaf - not included in this analysis if 'to some extent')

Currently housebound: Establish degree of mobility of subject (Usually ambulant nonhousebound, usually ambulant housebound, chairfast permanently, bedfast permanently).

* The MRC cognitive function and ageing study members were the following:

Professor T Arie, Professor J Bond, Dr C Brayne, Professor J Professor T Arie, Professor J Bond, Dr C Brayne, Professor J
Copeland, Professor N Day, Dr M Devakumar, Dr M E Dewey, Copeland, Professor N Day, Dr M Devakumar, Dr M E Dewey, sor J Grimley Evans, Dr F A Huppert, Ms J Illing, Dr A L Johnsor J Grimley Evans, Dr F A Huppert, Ms J Illing, Dr A L Johnson, Professor D W Kay, Mrs C McCracken, Mr M A McGee,
Professor I McKeith, Mrs D Mathewson, Dr K Morgan, Dr J Professor I McKeith, Mrs D Mathewson, Dr K Morgan, Dr J
Morris, Mrs J Nickson, Professor E S Paykel, Mrs C Parker, Mr G P Slegg, Mr N Walker.

We acknowledge with thanks the contribution of the local administrative and interviewing staff in the six study areas and the financial and other support given by the Medical Research Council.

Funding: Medical Research Council and Department of Health.

Conflicts of interest: none.

1 Office of Population Censuses and Surveys. General household survey: 1992. London: HMSO, 1994.

2 Office of Population Censuses and Surveys. 1991 census. London: HMSO, 1993.

3 Jagger C, Clarke M, Cook AJ. Mental and physical health of elderly people: five-year follow-up of a total population. Age Ageing 1989; 18: 77-82.
4 Ebrahim S, Dallosso H, Morgan K, Bassey J, Fentem P, Arie $T$. Causes of ill health among a random sample of old and very old people: possibilities for prevention. $\mathcal{F} R$ Coll Physicians Lond 1988; 22: 105-7.

5 Bowling A, Farquhar M, Grundy E. Associations with changes in level of functional ability. Results from a follow-up survey at two and a half years of people aged 85 follow-up survey at two and a half years of people aged 85
years and over at baseline interview. Ageing and Society 1994; 14: 53-73.

6 Jorm AF. The epidemiology of Alzheimer's disease and related disorders. London; Chapman and Hall, 1990.

7 Van Duijn CM, Stijnen T, Hofman A. Risk factors for Alzheimer's disease: overview of the EURODEM collaborative re-analysis of case-contrcl studies. Int $\mathcal{F}$ Epidemiology 1991; suppl 2: S4-12.

8 The MRC CFA Study. Design, Methodology and Baseline Sample. Psychol Med 1997. In press

9 Saunders PA, Copeland JRM, Dewey ME et al. The prevalence of dementia, depression and neurosis in later life: the Liverpool MRC-ALPHA study. Int f Epidemiology 1993; 22:838-47.

10 Office of Population Censuses and Surveys. Health survey for England 1992. London: HMSO, 1994.

11 Cox BD, Blaxter M, Buckle ALJ et al. The health and lifestyle survey. London; Health Promotion Research Trust, 1987.

12 Office of Population Censuses and Surveys. Surveys of disability in Great Britain: Report 1. London: HMSO, 1988.

13 Forsgren L. Prevalence of epilepsy in adults in northern Sweden. Epilepsia 1992;33:450-58.

14 Huijsman R. Economic evaluation of care for the chronically ill. Eur $\mathcal{f}$ Public Health 1995; 5: 8-19.

15 National Diabetes Data Group. Diabetes in America. Bethesda: National Institutes of Health, 1985.

16 Davis AC. The prevalence of hearing impairment and reported hearing disability in adults in Great Britain. Int $\mathcal{f}$ Epidemiol 1989; 18: 911-17.

17 The MRC CFA Study. Survey research into health problems of elderly people: a comparison of self-report with proxy information. Int $\mathcal{f}$ Epidemiol 1997. In press. 